\title{
Effects of modifiable prehospital factors on survival after out-of-hospital cardiac arrest in rural versus urban areas
}

\author{
Wenche Torunn Mathiesen ${ }^{1,27^{*}}$ (D), Conrad Arnfinn Bjørshol ${ }^{3}$, Jan Terje Kvaløy $y^{5,6}$ and Eldar Søreide ${ }^{1,4}$
}

\begin{abstract}
Background: The modifiable prehospital system factors, bystander cardiopulmonary resuscitation (CPR), emergency medical services (EMS), response time, and EMS physician attendance, may affect short- and long-term survival for both rural and urban out-of-hospital cardiac arrest (OHCA) patients. We studied how such factors influenced OHCA survival in a mixed urban/rural region with a high survival rate after OHCA.

Methods: We analyzed the association between modifiable prehospital factors and survival to different stages of care in 1138 medical OHCA patients from an Utstein template-based cardiac arrest registry, using Kaplan-Meier type survival curves, univariable and multivariable logistic regression and mortality hazard plots.

Results: We found a significantly higher probability for survival to hospital admission (OR: 1.84, 95\% Cl 1.43-2.36, $p<0.001$ ), to hospital discharge (OR: 1.51,95\% Cl 1.08-2.11, $p=0.017$ ), and at 1 year (OR: 1.58, 95\% Cl 1.11-2.26, $p=0.012$ ) in the urban group versus the rural group. In patients receiving bystander CPR before EMS arrival, the odds of survival to hospital discharge increased more than threefold (OR: 3.05, 95\% Cl 2.00-4.65, $p<0.001$ ).

However, bystander CPR was associated with increased patient survival to discharge only in urban areas (survival probability 0.26 with CPR vs. 0.08 without $C P R, p<0.001$ ). EMS response time $\geq 10$ min was associated with decreased survival (OR: $0.61,95 \% \mathrm{Cl} 0.45-0.83, p=0.002$ ), however, only in urban areas (survival probability $0.15 \geq 10$ min vs. 0.25 $<10$ min, $p<0.001$ ). In patients with prehospital EMS physician attendance, no significant differences were found in survival to hospital discharge (OR: 1.37, 95\% Cl 0.87-2.16, $p=0.17$ ). In rural areas, patients with EMS physician attendance had an overall better survival to hospital discharge (survival probability 0.17 with EMS physician vs. 0.05 without EMS physician, $p=0.019$ ). Adjusted for modifiable factors, the survival differences remained.

Conclusions: Overall, OHCA survival was higher in urban compared to rural areas, and the effect of bystander CPR, EMS response time and EMS physician attendance on survival differ between urban and rural areas. The effect of modifiable factors on survival was highest in the prehospital stage of care. In patients surviving to hospital admission, there was no significant difference in in-hospital mortality or in 1 year mortality between OHCA in rural versus urban areas.
\end{abstract}

Keywords: Out-of-hospital cardiac arrest, Rural, Urban, Survival, Cardiopulmonary resuscitation

\footnotetext{
* Correspondence: wenche.torunn.mathiesen@sus.no

${ }^{1}$ Critical Care and Anesthesiology Research Group, Stavanger University

Hospital, Stavanger, Norway

${ }^{2}$ Norwegian Air Ambulance Foundation, Department of Research and

Development, Drøbak, Norway

Full list of author information is available at the end of the article
} 


\section{Background}

Out-of-hospital cardiac arrest (OHCA) is a major cause of death in industrialized countries, and survival differs substantially, worldwide [1-3]. Survival is associated with certain OHCA patient factors, but these are generally non-modifiable [4]. However, some prehospital system factors in OHCA as bystander cardiopulmonary resuscitation (CPR), emergency medical services (EMS) response times, and the attendance of EMS physicians, are modifiable [4]. These factors represent opportunities for improvements in saving lives [4].

Bystander CPR is an essential intervention that increases the odds of survival of OHCA victims by two- to threefold [5]. As the effect of bystander CPR on survival declines rapidly over time, OHCA in remote areas with long EMS response times represent a particular challenge. Thus, a decrease in ambulance response times will lead to the annual saving of many additional lives [6]. In addition, some studies have shown that the attendance of an EMS physician is associated with improved survival $[7,8]$. Thus, it is likely that bystander CPR, EMS response times, and the attendance of an EMS physician influence short- and long-term survival. Because some studies have shown that also population density is a predictor for survival, [9-11] the aim of this study is to examine how bystander CPR, EMS response time, and EMS physician attendance influence survival at different stages of care, and to what extent this differs between rural and urban areas.

\section{Methods}

\section{Setting and design}

The study region was that encompassed by the Stavanger Hospital Trust; it is situated on the southwest coast of Norway and constitutes 18 municipalities. The area covers $5700 \mathrm{~km}^{2}$ and spans both urban and rural municipalities. A minority of the population are inhabitants on islands that can only be reached by boat or helicopter. The cities of Stavanger and Sandnes and two other municipalities are the most densely populated areas, with over 200 inhabitants per $\mathrm{km}^{2}$ (218-1733 inhabitants per $\left.\mathrm{km}^{2}\right)$. Geographically, these four municipalities constitute the Stavanger peninsula that, for the purpose of this study, constitutes the urban area. The other 14 municipalities have populations of less than 200 inhabitants per $\mathrm{km}^{2}$ (1-148 inhabitants per $\mathrm{km}^{2}$ ). In the present study, these municipalities constitute the rural area.

The population of the study area increased from approximately 300,000 inhabitants to approximately 358,000 inhabitants during the study period, from 1 January 2006 to 31 December 2015 [12]. In order to categorize rural versus urban municipalities, we used the mean population density during the study period.
Stavanger University Hospital (SUH) is the only receiving hospital for OHCA patients in the study region. The Emergency Dispatch Centre (EDC) is responsible for coordinating 17 ambulance units allocated to eight ambulance stations, and one hospital-based, anesthesiologist-manned (EMS physician) rapid response unit that uses a helicopter for remote assignments, or a car for local assignments. The ambulance staff constitutes two crew members, of which at least one is an advanced life support-certified paramedic. In addition, the EDC dispatches general practitioners (GPs) on call in the local communities [13]. From October 2013, an EMS physician-manned fast response car was made available for a rescue helicopter team, but operates only in the closest proximity to the helicopter base. In addition, fire brigades equipped with automated external defibrillators are often dispatched by the EDC and operate as first responders.

The EDC has one nationwide alarm emergency telephone number (113). The dispatch is criteria-based (using the Norwegian index of emergency medical assistance). In cases of suspected cardiac arrest, the EDC instructs the caller to start CPR, including mouth-tomouth resuscitation if the bystander is trained in CPR. In the case of untrained bystanders, the instruction is to carry out continuous chest compressions. The EDC also initiates a response by one or two ambulances, the EMS physician-manned rapid response unit and the local GP on call. The EDC aims to ensure at least one physician and two ambulance units at the scene. There were no systematic changes in the response pattern throughout the study period, except for the rapid response car of the rescue helicopter team. In cases where resuscitation did not lead to the return of spontaneous circulation (ROSC), the OHCA assignments were terminated at the scene or the patient was transported to hospital with ongoing resuscitation.

In Norway, training in basic CPR is provided through school systems, compulsory military service and voluntary organizations [14]. The oil industry employs a major work force in the Stavanger area, so CPR has been a part of health, environmental and safety training for many inhabitants in the study region, and there are also an increasing number of public access defibrillators (PADs). However, the EDC does not have alerting routines for PAD locations. As a part of post resuscitation intensive care at $\mathrm{SUH}$, an established in-hospital treatment for all OHCA patients who have not regained consciousness after hospital admission is targeted temperature management and standardized post resuscitation care $[15,16]$. Throughout the entire study period, emergency percutaneous coronary intervention was available to $\mathrm{OHCA}$ patients with ST-elevation myocardial infarction. 


\section{Study population}

The local registry for all EMS-attended OHCA cases has been managed by SUH since 1996; Utstein template data are collected from the ambulance, the rapid response unit, and the EDC and cross checked [17]. The present study assessed all prospective datasets of individuals aged $\geq 18$ years, collected between 1 January 2006 and 31 December 2014. OHCA missions for 2015 were collected from the Norwegian National Advisory Unit on Prehospital Emergency Medicine (NAKOS). We used the 2015 Utstein Resuscitation Registry Templates for Out-of-Hospital Cardiac Arrest to register the most likely primary cause of OHCA, which includes cardiac and other medical causes [18]. The following groups were excluded from further analysis: patients for whom no resuscitation attempts were made by the EMS, patients with EMS- or first responderwitnessed $\mathrm{OHCA}$, patients with $\mathrm{OHCA}$ of non-medical origin, and cases with missing data. A total of 25 patients had achieved ROSC before EMS arrival, but with no registering of initial rhythm. Nine of these patients had been shocked prior to EMS arrival and were included. During the 10-year study period, 171 patients were admitted to hospital with ongoing resuscitation, of whom nine survived to discharge. Of 2141 EMSattended OHCA incidents, a total of 1138 patients met the inclusion criteria for analysis (Fig. 1). Survival was measured at the following stages of care: hospital admission, ED discharge, $24 \mathrm{~h}$ after hospital admission, hospital discharge, and at 1 year. To further elucidate the relationship between prehospital modifiable factors and outcome, we also calculated the hazard of mortality between each stage.

\section{Study outcomes}

The primary outcome was patient survival to hospital discharge. Secondary outcomes were survival to hospital admission, survival to emergency department (ED) discharge, survival to $24 \mathrm{~h}$ after hospital admission, and survival at 1 year after cardiac arrest.

\section{Study variables}

The patient characteristic variables were age, gender, initial cardiac arrest rhythm (categorized as non-shockable [asystole, pulseless electrical activity] and shockable [ventricular fibrillation, pulseless ventricular tachycardia]), medical cause of cardiac arrest (cardiac and noncardiac), ROSC (yes, no), location of cardiac arrest (at home, in public), municipality (urban, rural), whether the cardiac arrest was witnessed, not witnessed, CPR initiated (by bystanders [bystander defined as a person who is not responding as a part of an EMS]), first responders/EMS), EMS response time (the time interval in minutes from the call to the EDC until the first emergency vehicle arrived at the patient's location) $(<10 \mathrm{~min}$, $\geq 10 \mathrm{~min}$ ), EMS physician attendance (yes, no), survival to hospital admission (yes, no), survival to ED discharge (yes, no), survival to $24 \mathrm{~h}$ after hospital admission (yes, no), survival to hospital discharge (yes, no), and survival at 1 year after cardiac arrest (yes, no).

\section{Statistical analysis}

All data retrieved from the local hospital-managed OHCA registry were entered into a FilemakerPro6 database (Filemaker, Inc., Santa Clara, CA, USA) and via Microsoft Office ${ }^{\circ}$ Excel 2010 (Microsoft Corporation,

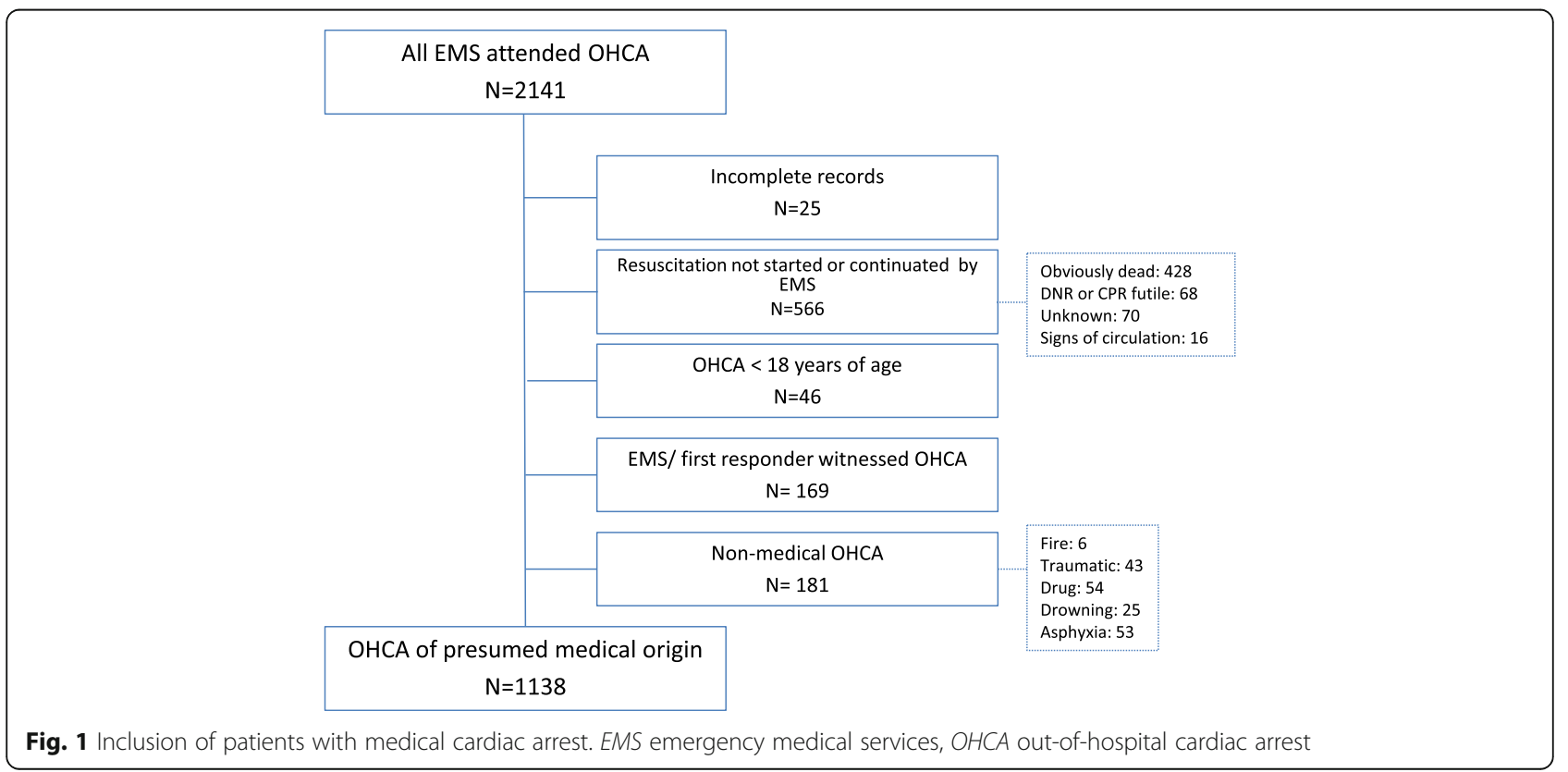


Table 1 Incidence, outcome and characteristics of medical out-of-hospital cardiac arrest patients $(n=1138)$

\begin{tabular}{|c|c|c|c|c|}
\hline & $\begin{array}{l}\text { Rural area } \\
(n=371)\end{array}$ & $\begin{array}{l}\text { Urban area } \\
(n=767)\end{array}$ & $p$,value & $\begin{array}{l}\text { Number of } \\
\text { missing data }\end{array}$ \\
\hline OHCA incidence/100,000/ year (adjusted rate) & $49(52)$ & $47(56)$ & 0.45 & \\
\hline Survival to hospital discharge, $\mathrm{n}(\%)$ & $55(14.8)$ & $159(20.7)$ & 0.021 & 0 \\
\hline Median patient age in years, (IQR) & $69(56-80)$ & $70(58-81)$ & 0.31 & 1 \\
\hline Median EMS response time in minutes, (IQR) & $11(7-16)$ & $9(7-12)$ & $<0.001$ & 0 \\
\hline Male gender, n (\%) & $263(71)$ & $522(68)$ & 0.37 & 0 \\
\hline Attended by EMS physician, n (\%) & $308(83)$ & $658(86)$ & 0.20 & 3 \\
\hline Shockable rhythm, n (\%) & $129(36)$ & $310(41)$ & 0.11 & 26 \\
\hline Prehospital ROSC, n (\%) & $111(30)$ & $286(37)$ & 0.017 & 0 \\
\hline Witnessed OHCA, n (\%) & $258(70)$ & $528(70)$ & 0.75 & 14 \\
\hline Bystander CPR, n (\%) & $267(73)$ & $537(71)$ & 0.39 & 11 \\
\hline Cardiac arrest location home, $\mathrm{n}(\%)$ & $229(62)$ & $508(66)$ & 0.17 & 1 \\
\hline Survival to hospital discharge in bystander witnessed OHCA with shockable first rhythm & $43(41)$ & $132(50)$ & 0.14 & 0 \\
\hline
\end{tabular}

The $p$ values are calculated by Poisson regression, The Mann-Whitney test, chi-square tests as appropriate

$C P R$ cardiopulmonary resuscitation, EMS emergency medical services, OHCA out-of-hospital cardiac arrest, ROSC return of spontaneous circulation

Redmond, WA, USA) to Statistical Package for the Social Sciences version 23 (IBM Corp, Armonk, NY,USA) and $R$ version 3.3.2 [19] for statistical analysis and plotting. Continuous variables are reported as medians and interquartile ranges (IQR), categorical variables as numbers and percentages. The Mann-Whitney test was used to test for difference in the distribution of continuous variables between the urban and rural groups. Chi-square tests for independence (with Yates' continuity correction) were used for assessing for differences in the distribution of categorical variables between groups. OHCA incidence rates were calculated, and age and gender adjusted according to the general Norwegian population, using data obtained from Statistics Norway [20]. Poisson regression analysis was used to test for differences in incidence rates between urban and rural areas. A Kaplan-Meier type plot was constructed to show estimated survival probabilities from after OHCA to five consecutive stages of care: Hospital admission, ED discharge, $24 \mathrm{~h}$ after admission, hospital discharge, and at 1 year. By focusing on these discrete stages of care rather than clock time we are able to do a detailed analysis of which factors are of importance for reaching these milestones. To further study the impact over time, hazard plots were also constructed. These plots show the estimated mortality probability between two consecutive stages of care, given survival to the start of the first stage. Chi-square tests were used to test for differences in mortality or hazards between groups for each stage of care. Logistic regression analysis was used to study the impact of urban and rural areas and other factors on survival to the different stages of care and at

Table 2 Odds ratios of key factors associated with survival

\begin{tabular}{|c|c|c|c|c|c|c|c|c|c|}
\hline & \multicolumn{3}{|c|}{$\begin{array}{l}\text { Survival to hospital } \\
\text { admission }\end{array}$} & \multicolumn{3}{|c|}{$\begin{array}{l}\text { Survival to hospital } \\
\text { discharge }\end{array}$} & \multicolumn{3}{|c|}{1 year survival } \\
\hline & $\mathrm{OR}$ & $95 \% \mathrm{Cl}$ & $p$ & $\mathrm{OR}$ & $95 \% \mathrm{Cl}$ & $p$ & OR & $95 \% \mathrm{Cl}$ & $p$ \\
\hline Urban vs. rural & 1.84 & $1.43-2.36$ & $<0.001$ & 1.51 & $1.08-2.11$ & 0.017 & 1.58 & $1.11-2.26$ & 0.012 \\
\hline EMS response time $\geq 10 \mathrm{~min}$ vs $<10 \mathrm{~min}$ & 0.69 & $0.55-0.87$ & 0.002 & 0.61 & $0.45-0.83$ & 0.002 & 0.57 & $0.41-0.79$ & $<0.001$ \\
\hline Age (one additional year) & 0.97 & $0.97-0.98$ & $<0.001$ & 0.96 & $0.95-0.97$ & $<0.001$ & 0.96 & $0.95-0.97$ & $<0.001$ \\
\hline Gender, male vs. female & 1.94 & $1.51-2.51$ & $<0.001$ & 3.15 & $2.10-4.72$ & $<0.001$ & 3.02 & $1.98-4.61$ & $<0.001$ \\
\hline EMS physician attendance vs. no EMS-physician attendance & 2.63 & $1.86-3.74$ & $<0.001$ & 1.37 & $0.87-2.16$ & 0.17 & 1.39 & $0.86-2.24$ & 0.18 \\
\hline Witnessed arrest vs. non-witnessed arrest & 4.12 & $3.12-5.44$ & $<0.001$ & 7.23 & $4.20-12.43$ & $<0.001$ & 6.63 & $3.78-11.61$ & $<0.001$ \\
\hline OHCA location in public vs. home & 1.20 & $1.10-1.30$ & $<0.001$ & 1.31 & $1.20-1.43$ & $<0.001$ & 1.27 & $1.16-1.39$ & $<0.001$ \\
\hline Bystander CPR vs. no bystander CPR & 1.98 & $1.52-2.58$ & $<0.001$ & 3.05 & $2.00-4.65$ & $<0.001$ & 2.84 & $1.83-4.39$ & $<0.001$ \\
\hline Shockable vs. non-shockable rhythm & 8.25 & $6.21-10.95$ & $<0.001$ & 25.74 & $15.71-42.18$ & $<0.001$ & 39.52 & $21.14-73.87$ & $<0.001$ \\
\hline Cardiac vs. medical cause for cardiac arrest & 1.31 & $0.92-1.88$ & 0.14 & 0.34 & $0.18-0.63$ & $<0.001$ & 0.16 & $0.07-0.40$ & $<0.001$ \\
\hline
\end{tabular}




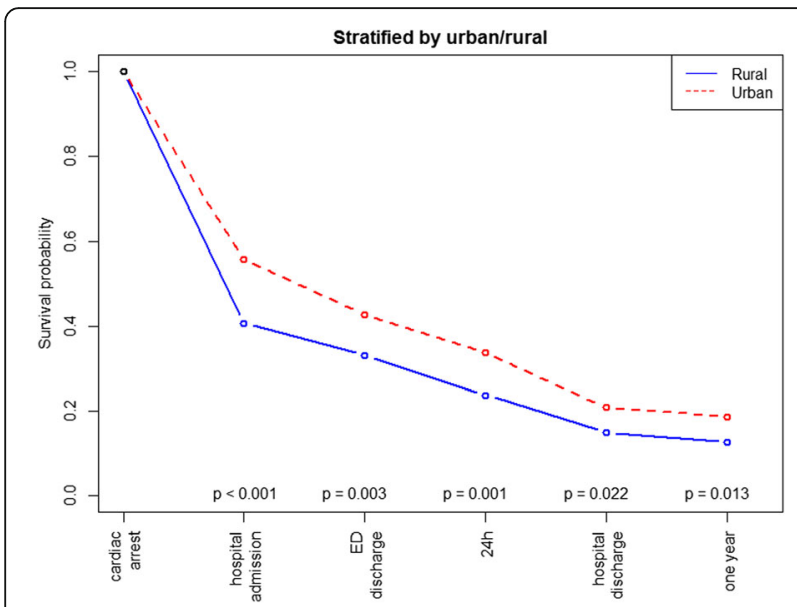

Fig. 2 Kaplan Meier type survival curves for out-of-hospital cardiac arrest patients in rural versus urban areas. ED emergency department

1 year. In all tests, $p$ values of $<0.05$ were considered statistically significant.

\section{Results}

The overall patient survival to hospital discharge was 18 . $8 \%$ and the unadjusted survival was higher in urban than in rural areas (Table 1). This was also reflected in the crude analysis with a significantly higher probability of survival to hospital admission (OR: 1.84, 95\% CI 1.43-2. $36, p=<0.001$ ), to hospital discharge (OR: $1.51,95 \%$ CI 1 . 08-2.11, $p=0.017$ ), and at 1 year (OR: $1.58,95 \%$ CI 1 . $11-2.26, p=0.012)$ in the urban group compared with the rural group (Table 2, Fig. 2). Bystander CPR was associated with improved survival to discharge only in urban areas (Fig. 3, $p<0.001$ ). EMS response times $>10$ min was associated with decreased survival to discharge only in urban areas (Fig. 4, $p<0.001$ ). The attendance by an EMS physician was associated with improved survival to hospital discharge only in rural areas (Fig. 5). When adjusted for the modifiable factors listed above, the difference in survival to hospital admission, hospital discharge, and at
1 year between rural and urban areas remained (Table 3). There was a significant higher prehospital hazard of death in OHCA victims in rural areas. However, if they survived to hospital admission, this hazard difference disappeared (Fig. 6).

\section{Discussion}

We conducted this retrospective analysis of registry data including 1138 OHCA patients with attempted resuscitation in a region where a high survival rate has previously been reported [14]. We found a significantly higher probability of survival to hospital admission, to hospital discharge, and at 1 year in the urban group compared with the rural group of patients. While bystander CPR and EMS response time $<10$ min were associated with a favorable outcome in urban patients at all stages of care, the attendance of an EMS physician was not. When adjusted for the modifiable factors; bystander CPR, attendance of EMS physician and EMS response time, the significant difference in survival to hospital admission, to hospital discharge, and at 1 year between rural and urban OHCA patients remained.

The present study shows a strong association between bystander CPR and survival, a finding that has been confirmed in several previous studies $[5,21]$. However, the current study also indicates that bystander CPR had a significant impact on survival in urban areas only. This major finding cannot be explained by differences in initial cardiac rhythm, gender, OHCA location, witnessed cardiac arrest, or bystander CPR rate between rural and urban areas. One reason could be lack of statistical power to detect a significant effect of bystander CPR in the rural group as there are few patients alive to hospital discharge. However, when assessing this finding, context characteristics must be considered. The social structure of rural communities means that there are fewer potential CPR bystanders compared with more densely populated areas. In urban areas, a greater number of potential bystanders are present, or in proximity, to initiate
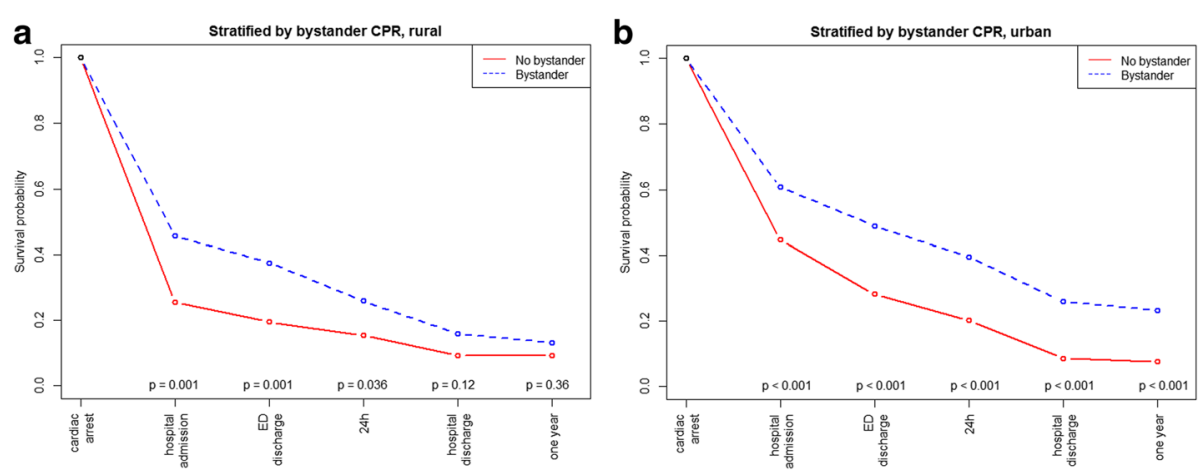

Fig. 3 Kaplan Meier type survival curves for out-of-hospital cardiac arrest patients stratified by bystander cardiopulmonary resuscitation in rural versus urban areas. CPR cardiopulmonary resuscitation, ED emergency department 

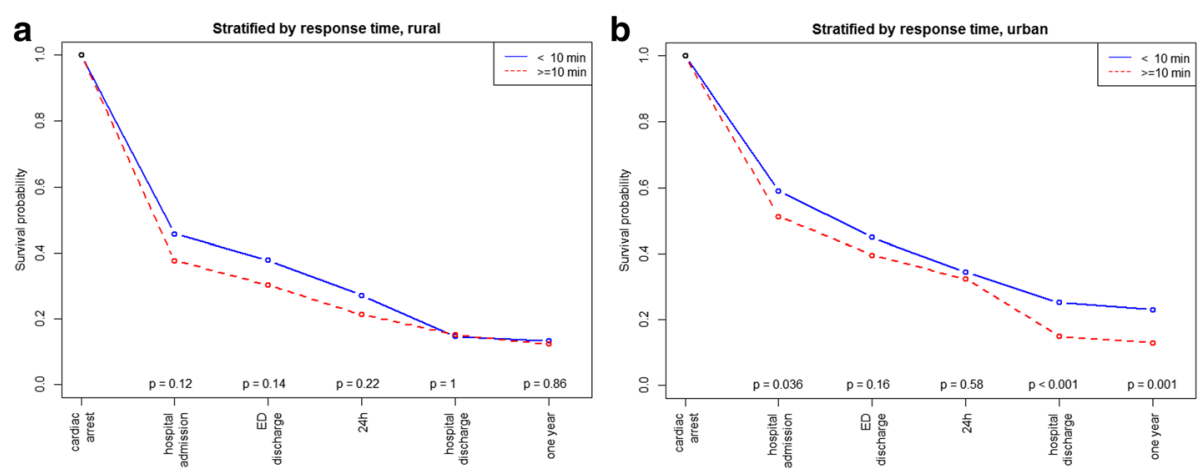

Fig. 4 Kaplan-Meier type survival curves for out-of-hospital cardiac arrest patients stratified by emergency medical services response time in rural versus urban areas. $E D$ emergency department

efficient interventions, such as calling the EDC and providing $\mathrm{CPR}$ in OHCA incidents. This may affect the quality of the handling of the OHCA incident before the EMS' arrival.

In the present study, the median EMS response time was 2 min shorter for the urban group than for the rural group. Considering the rapidly declining effect of bystander CPR with time, the lack of statistical significance between survival and EMS response time $<10 \mathrm{~min}$ in rural areas was surprising (Fig. 4) [6]. However, as time to intervention is crucial for survival, longer EMS response times may still explain the lower survival rate in the rural group. Optimizations in logistics can save lives, $[22,23]$ and EMS response time is thus one modifiable factor in improving survival.

Attendance by an EMS physician in OHCA was associated with overall improved survival at in-hospital stages of care and at 1 year in rural areas, but only with survival to hospital admission for OHCA in the urban group. Potential selection bias for EMS physician attendance at OHCA incidents may partially explain its association with short-term survival. Also, the high OR for survival to hospital discharge in rural areas may indicate a selection bias where EMS physician is called to certain types of OHCA. Some studies have been inconclusive or not shown a survival benefit of EMS physician attendance in OHCA [24, 25],. Hamilton et al. found an association between EMS physician attendance and both ROSC at the scene and 30-day survival [7]. The attendance by EMS physicians might have the greatest impact after ROSC has been achieved [26], and for the introduction of new treatment modalities, for example extracorporeal membrane oxygenation [27].

The higher OHCA survival in urban compared to rural areas found in the present study corresponds with the results of previous reports [9-11]. Still, among patients admitted alive, our results indicate no significant difference in later mortality, whether in the emergency department, $24 \mathrm{~h}$ after hospital admission, mortality at hospital discharge, or at 1 year. This is an important finding, and to our knowledge not reported before. It implies that further overall improvement in survival in rural areas must be based on community-based interventions [28]. Survival following rural OHCA could be improved by strengthening rural communities' CPR training [29], increase the use of dispatcher-assisted CPR [30], implement first-responder programs [31], provision of public access defibrillators [32], and optimize the
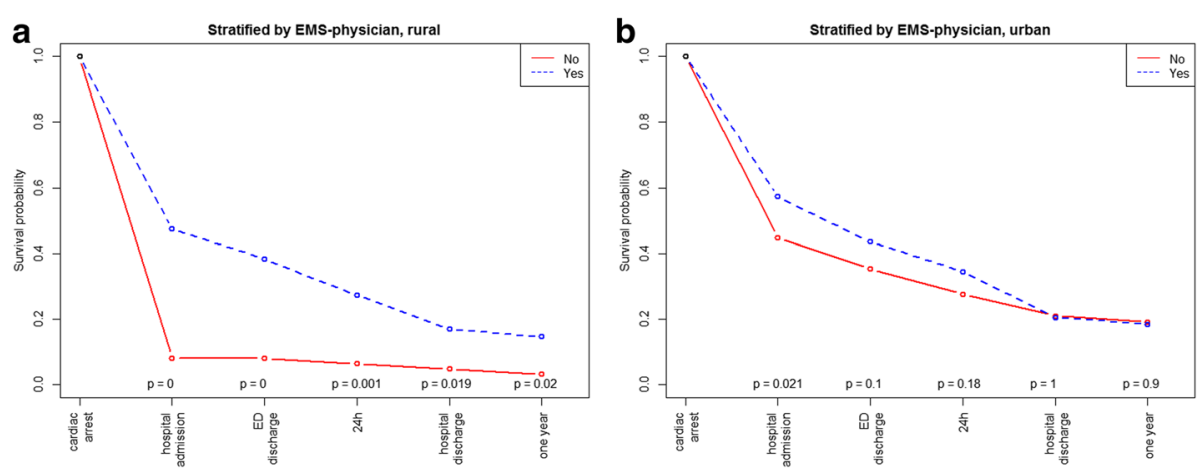

Fig. 5 Kaplan-Meier type survival curves for out-of-hospital cardiac arrest patients, stratified by emergency medical services physician attendance in rural versus urban areas. ED emergency department, EMS emergency medical services 
Table 3 Adjusted odds ratios for survival to different stages of care using geographical area and modifiable factors in the adjustments

\begin{tabular}{|c|c|c|c|c|c|c|c|c|c|}
\hline & \multicolumn{3}{|c|}{ Survival to hospital admission } & \multicolumn{3}{|c|}{ Survival to hospital discharge } & \multicolumn{3}{|c|}{ 1-year survival } \\
\hline & $\overline{\mathrm{OR}}$ & $95 \% \mathrm{Cl}$ & $p$ & OR & $95 \% \mathrm{Cl}$ & $p$ & OR & $95 \% \mathrm{Cl}$ & $p$ \\
\hline OHCA in urban area vs. rural area & 9.28 & $3.42-25.21$ & $<0.001$ & 5.11 & $1.45-18.05$ & 0.011 & 6.81 & $1.52-30.49$ & 0.012 \\
\hline Bystander CPR vs. no bystander CPR & 2.02 & $1.54-2.65$ & $<0.001$ & 2.98 & $1.95-4.56$ & $<0.001$ & 2.76 & $1.77-4.29$ & $<0.001$ \\
\hline EMS response time $\geq 10 \mathrm{~min} v \mathrm{vs} . \leq 10 \mathrm{~min}$. & 0.69 & $0.54-0.88$ & 0.003 & 0.61 & $0.44-0.84$ & 0.002 & 0.57 & $0.41-0.80$ & $<0.001$ \\
\hline $\begin{array}{l}\text { EMS physician attendance in rural area vs. no EMS } \\
\text { physician attendance in rural area }\end{array}$ & 10.7 & $3.94-26.25$ & $<0.001$ & 3.58 & $1.07-12.01$ & 0.039 & 4.71 & $1.10-20.15$ & 0.037 \\
\hline $\begin{array}{l}\text { EMS physician attendance in urban area vs. no EMS } \\
\text { physician attendance in urban area }\end{array}$ & 1.62 & $1.06-2.48$ & 0.025 & 0.91 & $0.54-1.45$ & 0.736 & 0.91 & $0.53-1.56$ & 0.730 \\
\hline
\end{tabular}

Adjusted odds ratios (multivariable analysis) of modifiable factors associated with survival to different stages of care in rural and urban out-of-hospital cardiac arrest $(\mathrm{OHCA})(\mathrm{n}=1138)$. Due to significant interaction with area EMS-physician are reported separately for urban and rural area

$\mathrm{Cl}$ confidence interval, $C P R$ cardiopulmonary resuscitation, EMS emergency medical services, $O R$ odds ratio

localization of EMS units [23]. With implementation of best practice, survival following OHCA could also be improved [33].

In the present study, bystander-witnessed OHCA with shockable first rhythm was $48.3 \%$ survival. Compared to a previous study conducted in the Stavanger region, in which a $52 \%$ survival to hospital discharge rate was found, the survival rate in the present study remains very good (Table 1) [14]. However, unlike the previous study, the results in the current study are based on non-EMS witnessed OHCA patients with a presumed medical cause (including a cardiac cause) for OHCA. Although the patient populations of the two studies are not entirely comparable, the results in the current study imply that the survival rate in the Stavanger region has not improved during the last decade, which contrasts with what has been found in several other regions [34, 35]. Opportunities for improvements include shortening of EMS response times, and strengthening community preparedness by e.g. additional lay person first responders via short message service alert or mobile app-based alert system. [36] To save more lives following OHCA, continuous endeavors to optimize modifiable factors are required to improve every link in the chain of survival.

\section{Limitations}

The data in this study did not allow us to assess patient neurological status and recovery in the surviving patients. However, previous studies from the Stavanger region have shown a good neurological status for the surviving patients [14], and there have been no major changes in OHCA patient treatment to indicate that this has changed over time. We chose the population and EMS response time categories that were appropriate for our region, so this choice may not be generalizable to other regions and countries. Further, we did not record the presence or absence of dispatcher-assisted CPR, which has been shown to affect survival in OHCA [37]. The calculated incidence of 47 OHCA per 100,000 per year in our region

Stratified by urban/rural

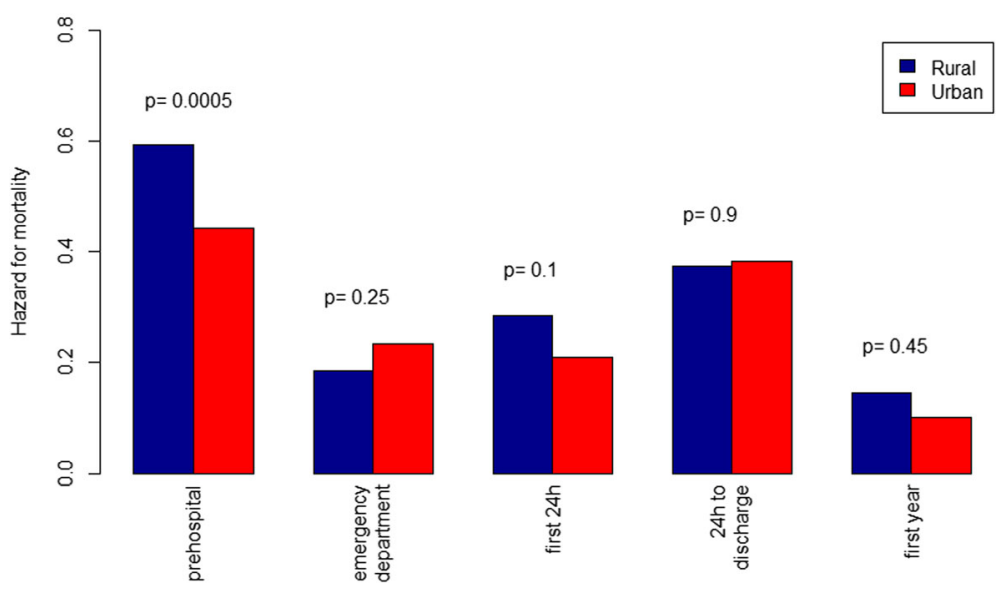

Fig. 6 Bar graphs illustrating the hazard for mortality in out-of-hospital cardiac arrest patients in rural versus urban areas. The hazard is the probability of dying between two consecutive stages of care, given that the patient is alive at the first stage 
is low compared to other reports [38], but the adjusted rate of 54 OHCA per 100,000 per year is according to OHCA in the general Norwegian population [39]. Also, for quality assurance, a designated nurse cross-checked data before entering into the OHCA database. Thus, we do not suspect selective reporting. The study region was that encompassed by the Stavanger Hospital Trust and the current findings may therefore not be generalizable to other different systems. Several hypothesis tests are conducted without any explicit adjustment for multiple testing. We acknowledge that some small $p$ values might have been obtained by chance, and in particular should $p$ values close to 0.05 be interpreted with care.

\section{Conclusions}

We found that OHCA survival was higher in urban compared to rural areas, and that the impact of the prehospital modifiable factors bystander CPR, EMS response time, and EMS physician attendance differed in urban and rural areas. The main difference is due to a lower ROSC rate and hospital admission rate of OHCA patients in rural areas. Importantly, in patients admitted alive to the hospital, survival rate did not differ between rural versus urban areas. Further improvements in survival in rural areas can be built on community-based interventions such as CPR training, first-responder programs, and public access defibrillation.

\section{Acknowledgements}

The authors thank the EMS at SUH for reporting OHCA data to the Regional Utstein Cardiac Registry. We also want to thank The Norwegian National Advisory Unit on Prehospital Emergency Medicine (NAKOS) for providing data.

\section{Funding}

The study was not funded.

\section{Availability of data and materials}

The data and materials used in the present study are available on request from the corresponding author.

\section{Authors' contributions \\ WTM contributed to the creation of the study material, participated in the design of the study, drafted and helped to revise the manuscript. CAB participated in the design of the study and helped to revise the manuscript. JTK participated in the design of the study, performed the statistical analyses and helped to revise the manuscript. ES contributed to the creation of the study material and helped to revise the manuscript. All authors read and approved the final manuscript and take full responsibility for all aspects of the study.}

\section{Ethics approval and consent to participate}

The Utstein template database was nationally and locally approved for scientific purposes by the Regional committee for Research Ethics and the Institutional Review Board at Stavanger Hospital Trust, Norway, respectively. Patient's informed consent was not considered necessary for this study to be approved as the purpose for the analysis of the data collection was for quality management.

\section{Consent for publication}

Not applicable.

\section{Competing interests}

WTM is employed with financial support by The Norwegian Air Ambulance Foundation.
$\mathrm{CAB}$ is employed by the Regional Competence Center for Acute Medicine in western Norway (RAKOS) with financial support from the Norwegian Directorate of Health. He has participated in Global Resuscitation Alliance meetings sponsored by the Laerdal Foundation for Acute Medicine, TrygFonden and EMS2017.

The authors declare that they have no competing interests.

\section{Publisher's Note}

Springer Nature remains neutral with regard to jurisdictional claims in published maps and institutional affiliations.

\section{Author details}

${ }^{1}$ Critical Care and Anesthesiology Research Group, Stavanger University Hospital, Stavanger, Norway. ${ }^{2}$ Norwegian Air Ambulance Foundation, Department of Research and Development, Drøbak, Norway. ${ }^{3}$ Department of Anesthesiology and Intensive Care, Stavanger University Hospital, Stavanger, Norway. ${ }^{4}$ Department of Clinical Medicine, University of Bergen, Bergen, Norway. ${ }^{5}$ Research Department, Stavanger University Hospital, Stavanger, Norway. ${ }^{6}$ Department of Mathematics and Physics, University of Stavanger, Stavanger, Norway. ${ }^{7}$ Stavanger University Hospital, Forskningens Hus, Armauer Hansensgate 2, P.O. box: 8100, 4068 Stavanger, Norway.

Received: 11 September 2017 Accepted: 21 March 2018

Published online: 18 April 2018

\section{References}

1. Nichol G, Thomas E, Callaway CW, Hedges J, Powell JL, Aufderheide TP, et al. Regional variation in out-of-hospital cardiac arrest incidence and outcome. JAMA. 2008;300:1423-31.

2. Atwood C, Eisenberg MS, Herlitz J, Rea TD. Incidence of EMS-treated out-ofhospital cardiac arrest in Europe. Resuscitation. 2005;67:75-80.

3. Girotra S, van Diepen S, Nallamothu BK, Carrel M, Vellano K, Anderson ML, et al. Regional variation in out-of-hospital cardiac arrest survival in the United States. Circulation. 2016;133(22):2159-68.

4. Bjørshol CA, Søreide E. Improving survival after cardiac arrest. Semin Neurol. 2017;37:25-32.

5. Sasson C, Rogers MA, Dahl J, Kellermann AL. Predictors of survival from outof-hospital cardiac arrest: a systematic review and meta-analysis. Circ Cardiovasc Qual Outcomes. 2010;3:63-81.

6. Rajan S, Wissenberg M, Folke F, Hansen SM, Gerds TA, Kragholm K, et al. Association of bystander cardiopulmonary resuscitation and survival according to ambulance response-times after out-of-hospital cardiac arrest. Circulation. 2016;134:2095-104.

7. Hamilton A, Steinmetz J, Wissenberg M, Torp-Pedersen C, Lippert FK, Hove $L$, et al. Association between prehospital physician involvement and survival after out-of-hospital cardiac arrest: a Danish nationwide observational study. Resuscitation. 2016;108:95-101.

8. Bottiger BW, Bernhard M, Knapp J, Nagele P. Influence of EMS-physician presence on survival after out-of-hospital cardiopulmonary resuscitation: systematic review and meta-analysis. Crit Care. 2016;20:4.

9. Nehme Z, Andrew E, Cameron PA, Bray JE, Bernard SA, Meredith IT, et al. Population density predicts outcome from out-of-hospital cardiac arrest in Victoria, Australia. Med J Aust. 2014;200:471-5.

10. Chen C-C, Chen C-W, Ho C-K, Liu IC, Lin B-C, Chan T-C. Spatial Variation and Resuscitation Process Affecting Survival after Out-of-Hospital Cardiac Arrests (OHCA). PLoS One. 2015;10:e0144882.

11. Hiltunen P, Kuisma M, Silfvast T, Rutanen J, Vaahersalo J, Kurola J, et al. Regional variation and outcome of out-of-hospital cardiac arrest (ohca) in Finland - the Finnresusci study. Scand J Trauma Resusc Emerg Med. 2012;20:80.

12. Statistics Norway. Available from: http://www.ssb.no/.

13. Langhelle A, Lossius HM, Silfvast T, Bjornsson HM, Lippert FK, Ersson A, et al. International EMS Systems: the Nordic countries. Resuscitation. 2004;61:9-21.

14. Lindner TW, Søreide E, Nilsen OB, Torunn MW, Lossius HM. Good outcome in every fourth resuscitation attempt is achievable- an Utstein template report from the Stavanger region. Resuscitation. 2011;82:1508-13.

15. Busch M, Soreide E, Lossius HM, Lexow K, Dickstein K. Rapid implementation of therapeutic hypothermia in comatose out-of-hospital cardiac arrest survivors. Acta Anaesthesiol Scand. 2006;50:1277-83.

16. Sunde K, Pytte M, Jacobsen D, Mangschau A, Jensen LP, Smedsrud C, et al. Implementation of a standardised treatment protocol for post resuscitation care after out-of-hospital cardiac arrest. Resuscitation. 2007;73:29-39. 
17. Cummins RO, Chamberlain DA, Abramson NS, Allen M, Baskett PJ, Becker L, et al. Recommended guidelines for uniform reporting of data from out-ofhospital cardiac arrest: the Utstein Style. A statement for health professionals from a task force of the American Heart Association, the European Resuscitation Council, the Heart and Stroke Foundation of Canada, and the Australian Resuscitation Council. Circulation. 1991;84:960-75.

18. Perkins GD, Jacobs IG, Nadkarni VM, Berg RA, Bhanji F, Biarent D, et al. Cardiac arrest and cardiopulmonary resuscitation outcome reports: update of the Utstein Resuscitation Registry Templates for out-of-hospital cardiac arrest: a statement for healthcare professionals from a task force of the International Liaison Committee on Resuscitation (American Heart Association, European Resuscitation Council, Australian and New Zealand Council on Resuscitation, Heart and Stroke Foundation of Canada, InterAmerican Heart Foundation, Resuscitation Council of Southern Africa, Resuscitation Council of Asia); and the American Heart Association Emergency Cardiovascular Care Committee and the Council on Cardiopulmonary, Critical Care, Perioperative and Resuscitation. Resuscitation. 2015;96:328-40.

19. RC Team. R: A language and environment for statistical computing. $R$ Foundation for Stastistical Computing: Vienna, Austria; 2016. https://www. gbif.org/tool/81287/r-a-language-and-environment-for-statistical-computing.

20. Statistics Norway. Available from: https://www.ssb.no/en/befolkning/ statistikker/folkemengde. Accessed 16 Jan 2017.

21. Hasselqvist-Ax I, Riva G, Herlitz J, Rosenqvist M, Hollenberg J, Nordberg P, et al. Early Cardiopulmonary Resuscitation in Out-of-Hospital Cardiac Arrest. N Engl J Med. 2015;372:2307-15.

22. Strömsöe A, Svensson L, Claesson A, Lindkvist J, Lundstrom A, Herlitz J. Association between population density and reported incidence, characteristics and outcome after out-of-hospital cardiac arrest in Sweden. Resuscitation. 2011;82:1307-13.

23. Strömsöe A, Afzelius S, Axelsson C, Sodersved Kallestedt ML, Enlund M, Svensson L, et al. Improvements in logistics could increase survival after out-of-hospital cardiac arrest in Sweden. J Intern Med. 2013;273:622-7.

24. Dyson K, Bray J, Smith K, Bernard S, Finn J. A systematic review of the effect of emergency medical service practitioners' experience and exposure to out-of-hospital cardiac arrest on patient survival and procedural performance. Resuscitation. 2014;85:1134-41.

25. Olasveengen TM, Lund-Kordahl I, Steen PA, Sunde K. Out-of hospital advanced life support with or without a physician: effects on quality of CPR and outcome. Resuscitation. 2009;80:1248-52.

26. Lyon RM, Nelson MJ. Helicopter emergency medical services (HEMS) response to out-of-hospital cardiac arrest. Scand J Trauma Resusc Emerg Med. 2013;21:1.

27. Schober A, Sterz F, Herkner H, Wallmueller C, Weiser C, Hubner P, et al. Emergency extracorporeal life support and ongoing resuscitation: a retrospective comparison for refractory out-of-hospital cardiac arrest. Emerg Med J. 2017;34(5):277-81. https://doi.org/10.1136/emermed-2015-205232.

28. Rea TD, Page RL. Community approaches to improve resuscitation after outof-hospital sudden cardiac arrest. Circulation. 2010;121:1134-40.

29. Moller Nielsen A, Lou Isbye D, Knudsen Lippert F, Rasmussen LS. Engaging a whole community in resuscitation. Resuscitation. 2012;83:1067-71.

30. Bobrow BJ, Spaite DW, Vadeboncoeur TF, Hu C, Mullins T, Tormala W, et al. Implementation of a regional telephone cardiopulmonary resuscitation program and outcomes after out-of-hospital cardiac arrest. JAMA Cardiol. 2016;1:294-302.

31. Nordberg P, Hollenberg J, Rosenqvist M, Herlitz J, Jonsson M, JarnbertPetterson $\mathrm{H}$, et al. The implementation of a dual dispatch system in out-ofhospital cardiac arrest is associated with improved short and long term survival. Eur Heart J Acute Cardiovasc Care. 2014;3:293-303.

32. Zijlstra JA, Stieglis R, Riedijk F, Smeekes M, van der Worp WE, Koster RW Local lay rescuers with AEDs, alerted by text messages, contribute to early defibrillation in a Dutch out-of-hospital cardiac arrest dispatch system. Resuscitation. 2014;85:1444-9.

33. Eisenberg MLF, Shin SD, et al. Improving survival from out-of-hospital cardiac arrest: a call to establish a global resuscitation alliance. Stavanger, Norway: Utstein Abbey; 2016. Available from: https://emseurope.org/wp-content/ uploads/2016/10/A-Call-to-Establish-a-Global-Resuscitation-Alliance1.pdf

34. Wissenberg M, Lippert FK, Folke F, Weeke P, Hansen CM, Christensen EF, et al. Association of national initiatives to improve cardiac arrest management with rates of bystander intervention and patient survival after out-of-hospital cardiac arrest. JAMA. 2013;310:1377-84

35. Fothergill RT, Watson LR, Chamberlain D, Virdi GK, Moore FP, Whitbread M. Increases in survival from out-of-hospital cardiac arrest: a five year study. Resuscitation. 2013;84:1089-92.
36. Caputo ML, Muschietti S, Burkart R, Benvenuti C, Conte G, Regoli F, et al. Lay persons alerted by mobile application system initiate earlier cardio-pulmonary resuscitation: a comparison with SMS- based system notification. Resuscitation. 2017;114:73-8

37. Lerner EB, Rea TD, Bobrow BJ, Acker JE, Berg RA, Brooks SC, et al. Emergency medical service dispatch cardiopulmonary resuscitation prearrival instructions to improve survival from out-of-hospital cardiac arrest. Circulation. 2012;125:648-55.

38. Berdowski J, Berg RA, Tijssen JG, Koster RW. Global incidences of out-of-hospital cardiac arrest and survival rates: Systematic review of 67 prospective studies. Resuscitation. 2010;81:1479-87.

39. Tjelmeland IBM, Jan Erik Nilsen JK-J, Andersson L-J, Bratland S, Haug B, Langørgen J, et al. Norsk hjertestansregister. Årsrapport for 2015 med plan for forbedringstiltak. Oslo: The Norwegian National Advisory Unit on Prehospital Emergency Medicine (NAKOS); 2016

\section{Submit your next manuscript to BioMed Central and we will help you at every step:}

- We accept pre-submission inquiries

- Our selector tool helps you to find the most relevant journal

- We provide round the clock customer support

- Convenient online submission

- Thorough peer review

- Inclusion in PubMed and all major indexing services

- Maximum visibility for your research

Submit your manuscript at www.biomedcentral.com/submit
C Biomed Central 